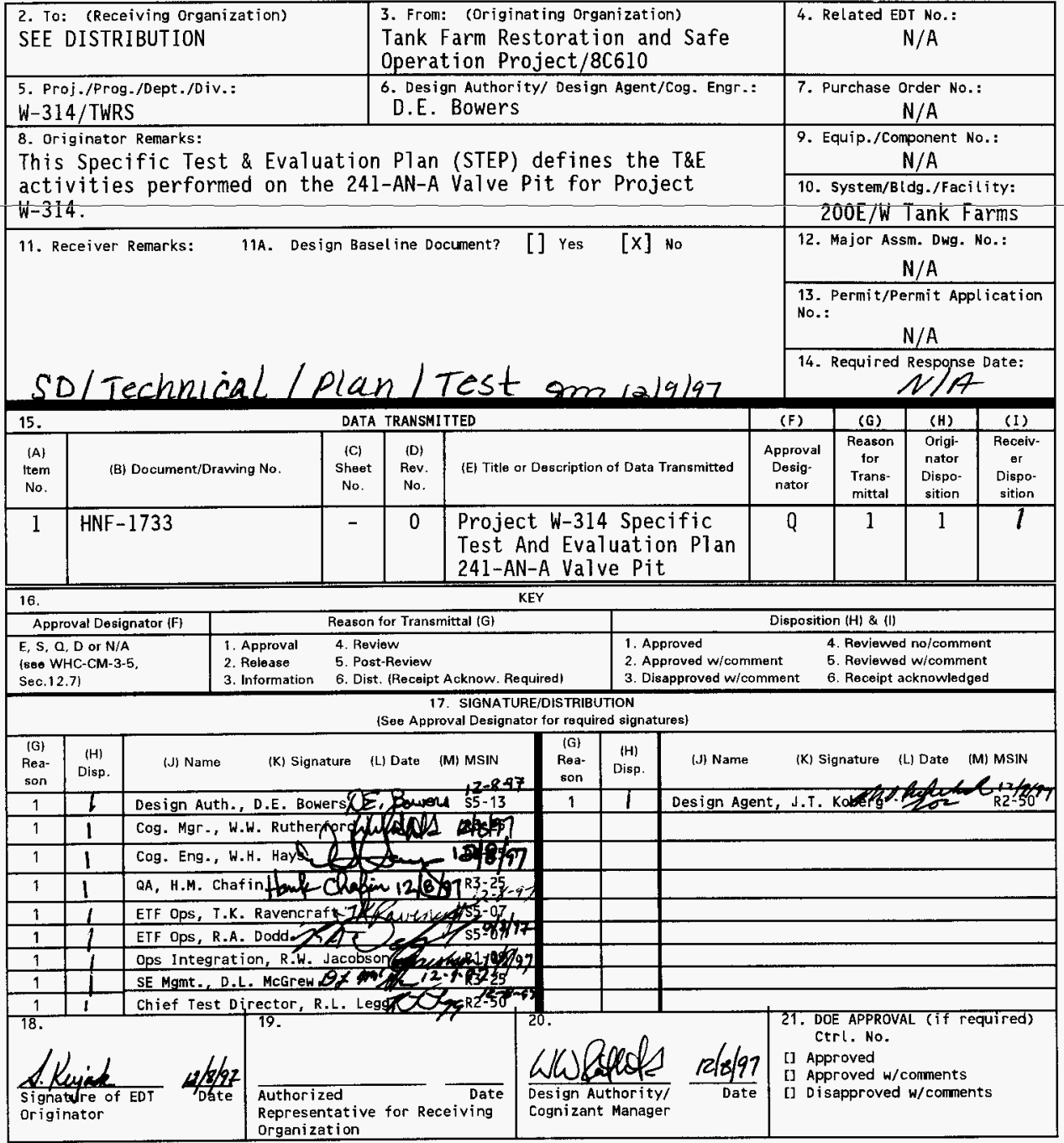

BD-7400-172-2 (05/96) GEF097 
HNF-1733, Rev. 0

\section{SPECIFIC TEST AND EVALUATION PLAN}

\section{William H Hays}

Numatec Hanford Company, Richland, WA 99352

U.S. Department of Energy Contract DE-AC06-96RL13200

$\begin{array}{lll}\text { EDT/ECN: } & 622787 & \text { UC: } 2030 \\ \text { Org Code: } & 86610 & \text { Charge Code: N314K } \\ \text { B\&R Code: } & \text { EW3130010 } & \text { Total Pages: 27 } 28 \mathrm{gm} \text { 12/9/97 }\end{array}$

Key Words: Specific Test and Evaluation Plan, Project W-314, 241-AN-A Valve Pit

Abstract: This Specific Test and Evaluation Plan (STEP) defines the test \& evaluation activities performed on the 24l-AN-A Valve Pit for the W-314 Project.

TRADEMARK DISCLAIMER. Reference herein to any specific commercial product, process, or service by trade name, trademark, manufacturer, or otherwise, does not necessarily constitute or imply its endorsement, recommendation, or favoring by the United States Government or any agency thereof or its contractors or subcontractors.

Printed in the United States of America. To obtain copies of this document, contact: Document Control Services, P.O. Box 950, Mailstop H6-08, Richland WA 99352, Phone (509) 372-2420;

Fax (509) 376-4989.

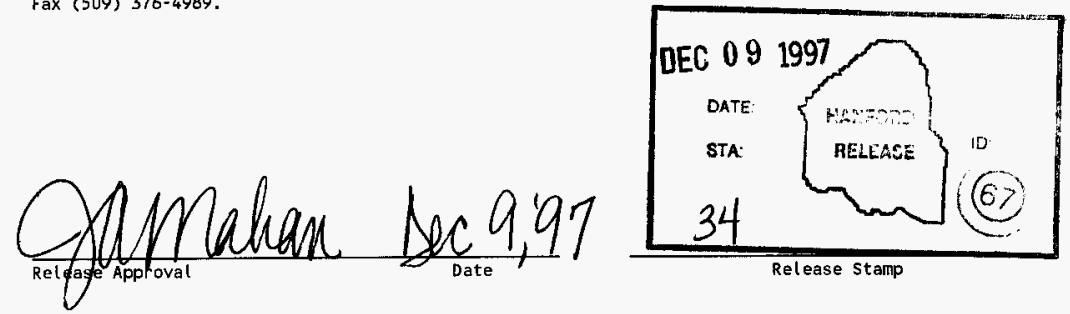

Approved for Public Release 
PROJECT W-314

\section{SPECIFIC TEST AND EVALUATION PLAN}

241-AN-A VALVE PIT 
1.0 PURPOSE

2.0 SCOPE

3.0 ADMINISTRATIVE

3.1 SAFETY INFORMATION/COMMUNICATIONS 4

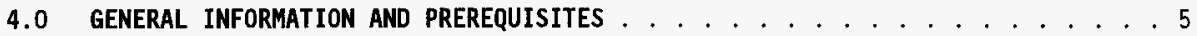

4.1 INITIAL CONDITIONS .................. 5

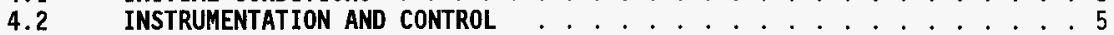

4.3 FUNCTIONAL TESTS . . . . . . . . . . . . . . . . . . . 5

4.4 TEMPORARY MODIFICATIONS . . . . . . . . . . . . . . . . . 5

4.5 EQUIPMENT AND MATERIALS ............... 5

5.0 ASSIGNMENTS OF RESPONSIBILITY . . . . . . . . . . . . . . . . . . . 5

5.1 JOINT TEST REVIEW GROUP . . . . . . . . . . . . . . 5

5.2 TEST CONFIGURATION CONTROL . . . . . . . . . . . . 6

6.0 SYSTEM BOUNDARIES . . . . . . . . . . . . . . . . 6

6.1 OBJECTIVE . . . . . . . . . . . . . . . 6

6.2 DESCRIPTION $\ldots \ldots \ldots$

6.3 SYSTEM TURNOVER . . . . . . . . . . . . . . . . . . . . . 11

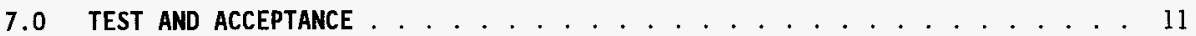

7.1 VERIFICATION \& VALIDATION ............... 11

7.2 COMMERCIAL GRADE ITEM DEDICATION . . . . . . . . . 12

7.3 FACTORY ACCEPTANCE TESTING . . . . . . . . . . . . . 12

7.3 CONSTRUCTION ACCEPTANCE TESTING . . . . . . . . . . . 13

7.4 ACCEPTANCE TESTING . . . . . . . . . . . . . . . 15

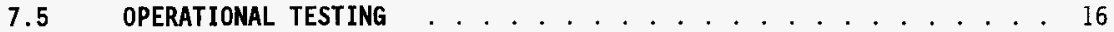

REFERENCES . . . . . . . . . . . . . . . . . . . . . . 18

241-AN-A VALVE PIT TEST COMPLETION CHECKLIST . . . . . . . . . . . . . 21 


\section{TABLE OF CONTENTS (CONT.)}

\section{LIST OF TABLES}

TABLE 5-1 241-AN-A VALVE PIT WORK AND TEST BOUNDARIES . . . . . . . . . 7

TABLE 7-2 COMMERCIAL GRADE ITEM DEDICATION ACTIVITIES . . . . . . . . . 12

TABLE $7-3$ FACTORY ACCEPTANCE TESTS . . . . . . . . . . . . . 12

TABLE $7-4$ CONSTRUCTION ACCEPTANCE TESTS . . . . . . . . . . . 13

TABLE $7-5$ ACCEPTANCE TEST PROCEDURES . . . . . . . . . . . 15

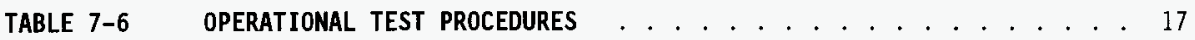

$$
\text { LIST OF FIGURES }
$$

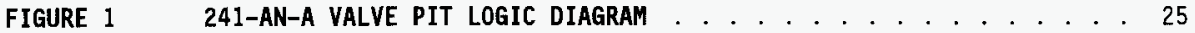


HNF-1733, REV. 0

\subsection{PURPOSE}

The purpose of this Specific Test and Evaluation Plan (STEP) is to provide a detailed written $\mathrm{plan}$ for the systematic testing of modifications made to the 24l-AN-A Valve Pit by the $W-314$ Project. The STEP develops the outline for test procedures that verify the system's performance to the established Project design criteria. The STEP is a "lower tier" document based on the W-314 Test \& Evaluation Plan (TEP).

\subsection{SCOPE}

This STEP encompasses all testing activities required to demonstrate compliance to the project design criteria as it relates to the modifications of the AN-A valve pit. The Project Design Specifications (PDS) identify the specific testing activities required for the Project. Testing includes Validations and Verifications (e.g., Commercial Grade Item Dedication activities...etc), Factory Acceptance Tests (FATs), installation tests and inspections, Construction Acceptance Tests (CATs), Acceptance Test Procedures (ATPS), Pre-0perational Test Procedures (POTPS), and 0perational Test Procedures (OTPS). It should be noted that POTPs are not required for testing of the modifications to the 241-AN-A Valve Pit. The STEP will be utilized in conjunction with the TEP for verification and validation.

\subsection{ADMINISTRATIVE}

\subsection{Safety Information/Communications}

Testing and inspection activities will be performed to the safety and communication procedures of the testing facility (e.g., shop, mockup, tank farm, vendor facility...etc.)

During the performance of 241-AN Tank Farm ATPs and 0TPs, the Operations Test Director will be the direct line of communication with the Shift office. During abnormal and casualty situations, the Double-She11 Tank Farms Shift Manager will be the building emergency director/event commander. 


\subsection{GENERAL INFORMATION AND PREREQUISITES}

\subsection{Initial Conditions}

Prerequisite tests and all required construction, specific to the test, shall be completed prior to beginning any test. Test boundaries shall be verified as being appropriate and secure. Required locks and tags shall be correctly placed to allow performance of the test without disturbing the lock and tag. Required personnel shall be available and briefed on the steps of the test (a pre-job briefing is a prerequisite that must be satisfied before performing a Construction Work Package (CWP), ATP, and/or OTP). Required test apparatus shall be functional and set up in a safe configuration for the test. Installed and existing equipment, required for the test, shall be verified to be ready to operate in the test conditions.

\subsection{Measurement \& Test Equipment}

Instruments used will be specified per the test document and controlled per the performing agency's Measurement \& Test Equipment program.

\subsection{Functional Tests}

Functional tests, when required for calibration of equipment, will be performed prior to the asSOciated ATP or 0TP. Functional tests will be performed to approved documents.

\subsection{Temporary Modifications}

Temporary modifications may be required in 241-AN Tank Farm. Temporary modifications will be made per HNF-IP-0842, Vol. IV, section 4.5 .

\subsection{Equipment and Materials}

Equipment installed by the project that fails during testing, or existing equipment that is damaged by testing, will be the responsibility of the construction contractor. Existing facility SSCS, not damaged but found defective, will be the responsibility of Double-She11 Tanks organization. Initiation of repairs will be accomplished by the creation of either a work package, using JCS, or a Construction Work Package (CWP).

\subsection{ASSIGNMENTS OF RESPONSIBILITY}

The design agent (FDNW) will provide test directors for the Acceptance Test Procedures. Tank Farm Operations will provide a qualified Tank Farm Shift Manager or 0perations Engineer who will be the Test Directors for all OTPs. This representative will accept the results of the ATPs for Tank Farm Operations. Roles and responsibilities for construction, TWRS Operations, TFRSO Startup Group, W-314 Project Management, Acceptance Inspection, and Quality Assurance are defined in the W-314 TEP, Chapter three. 


\subsection{Joint Test Review Group}

The JTRG will consist of the Chief Test Director (from the TWRS Testing and Systems Readiness organization), Engineering manager (of the facility that will operate the equipment), W-314 Startup Manager, the Design Authority, applicable Test Director for each ATP \& OTP, and W-314 Project Manager. The JTRG provides Tank Farm Operations with a high level of confidence that the test can be performed safely and efficiently. The review by the JTRG provides a basis for test procedure approval and release. The JTRG conducts thorough reviews of ATPs and 0TPs to ensure compliance with applicable procedural requirements, to ensure they can be performed safely, and to ensure the scope of testing and inspections provide a product that satisfies operational and safety requirements. The JTRG reviews test procedure data, and provides their recommendation concerning the final acceptance that test results satisfy the design specifications as stated in the ATPS, OTPs, or test plans.

\subsection{Test Configuration Control}

Testing shall be conducted using detailed test procedures and the latest versions of all ECNs and Drawings for the Project.

Configuration control shall conform to HNF-PRO-440 and HNF-PRO-226. Drawings shall be retained in project status by the $W-314$ Project until such time as all work on the 24l-AN-A valve pit is complete and accepted.

\subsection{SYSTEM BOUNDARIES}

\section{1 objective}

Specific system boundaries are identified for use in planning and implementing the various tests listed in this STEP. These system boundaries will provide safe work boundaries which allow testing to proceed in a safe environment, isolated from facility hazards.

\subsection{Administrative Controls}

Where actual physical isolations are not possible (e.g., pit nozzles...etc), Lock and Tag system (HNF-IP-0842, Vol. Il, Sections 4.9.1 and 4.10.1), as a minimum, will be utilized to establish the required administrative controls.

\subsection{Description}

The table below 1 ists the work and test boundaries for 241-AN-A valve pit work. These boundaries are estimates and may be modified at the time of execution to match current conditions in the farm and the job. This table will not be modified to reflect those changes. 
HNF-1733, REV. 0

TABLE 5-1 241-AN-A VALVE PIT WORK AND TEST BOUNDARIES

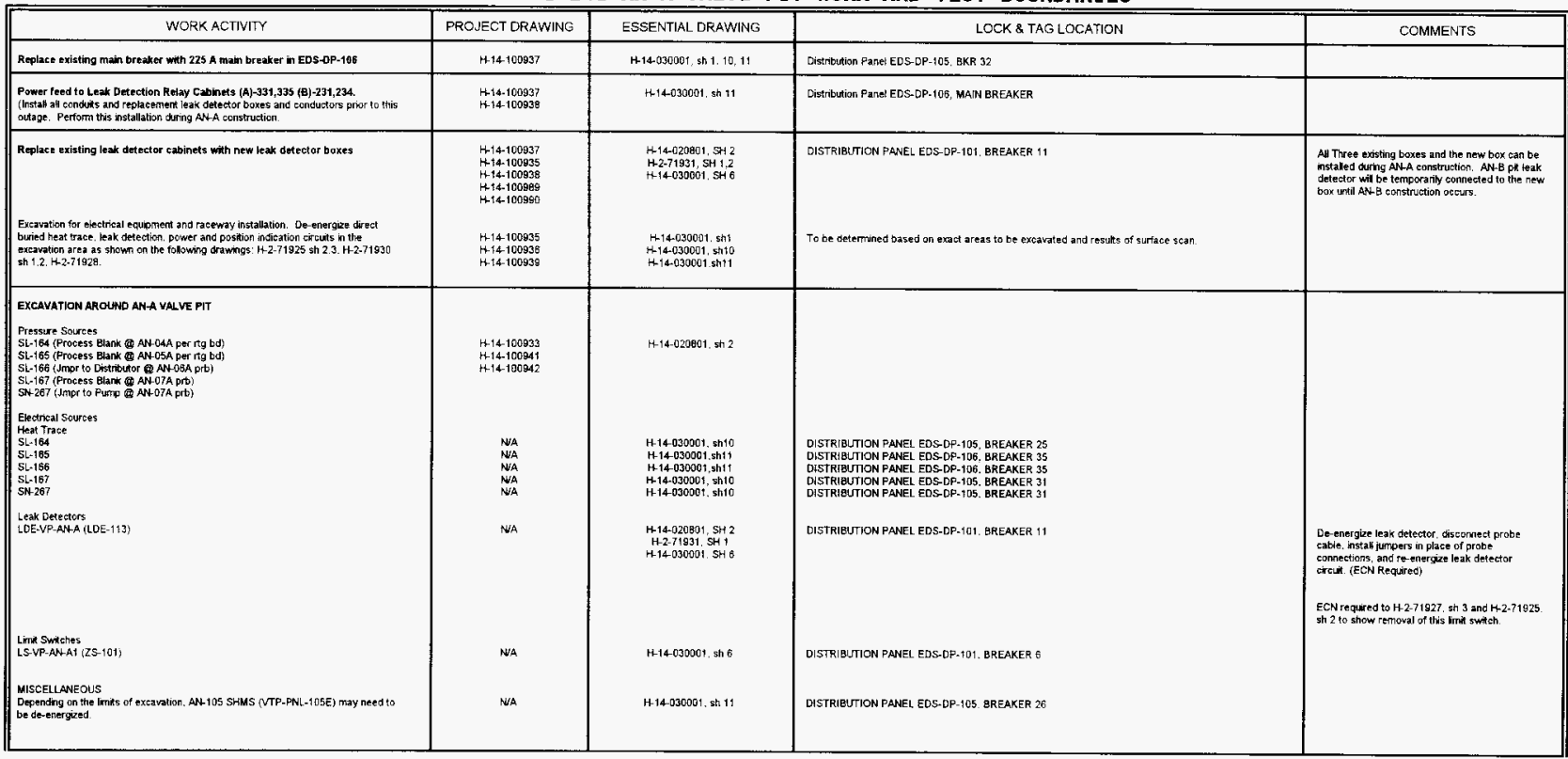




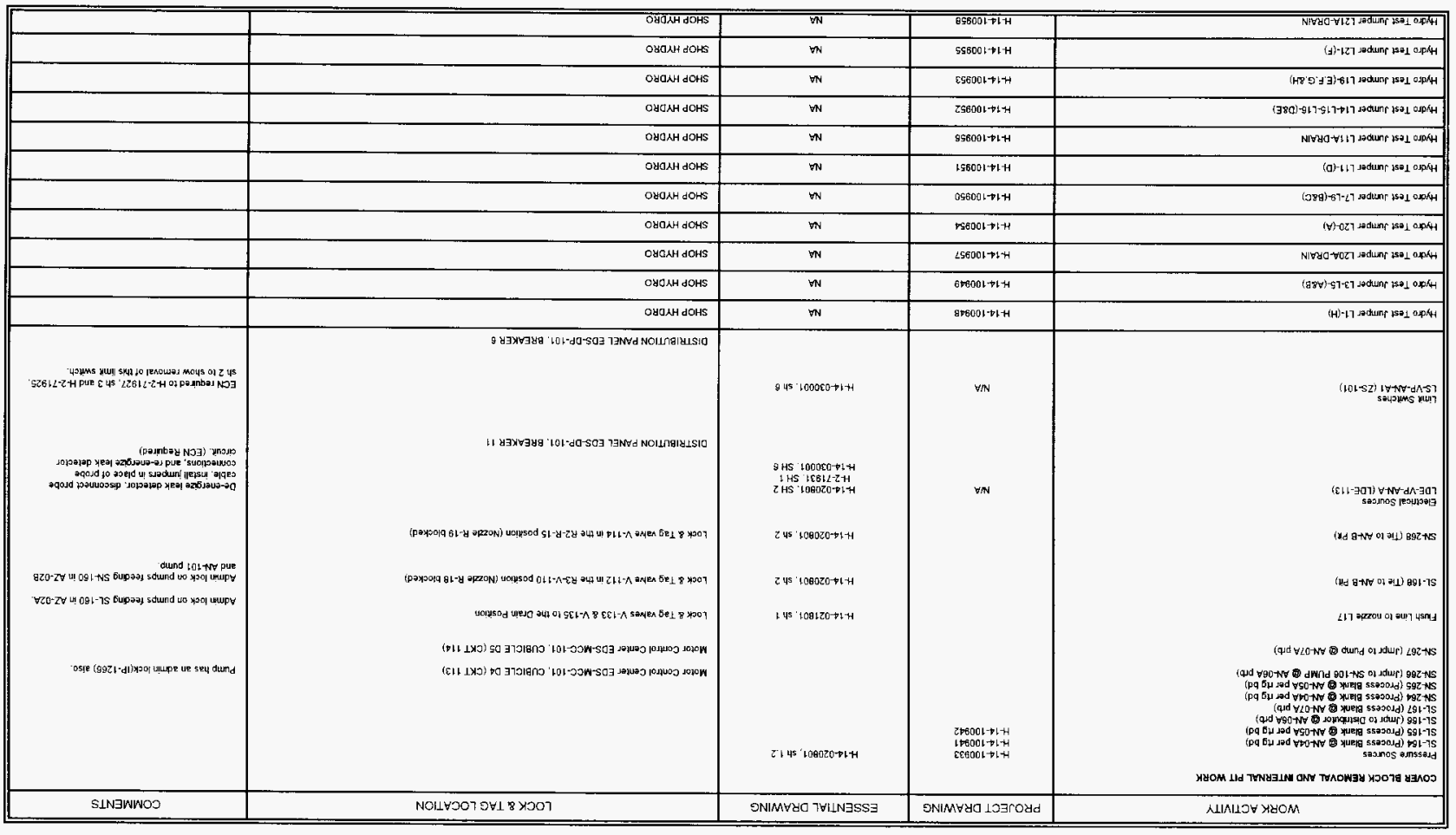

$0 \cdot A \exists y$ ' $\varepsilon \varepsilon \angle[ - \pm N H$ 


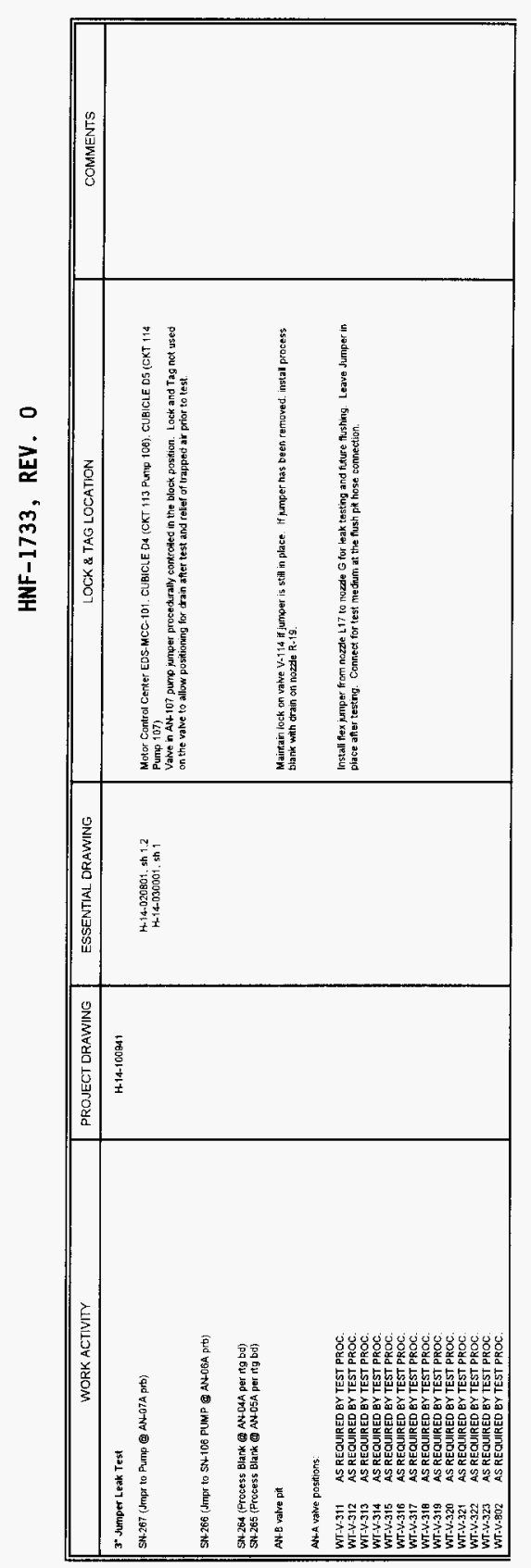




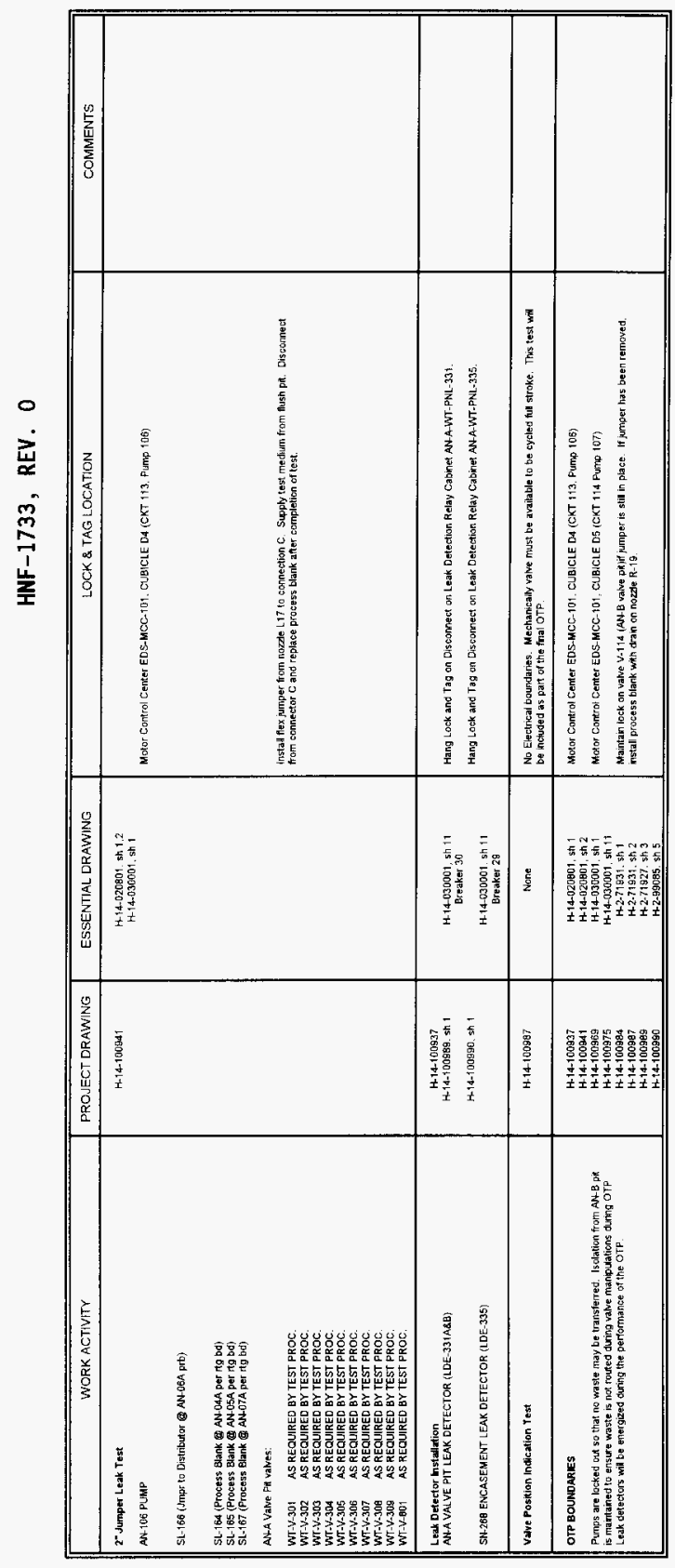

으 
HNF-1733, REV. 0

\subsection{System Turnover}

Following completion and acceptance of a 11 the required testing and testing data within a given boundary, the systems and equipment in that area will be ready for release to operations for their control and use. The SSC(s) will be released to TWRS Operations by using the Acceptance for Beneficial Use process described in the W-314 TEP (HNF-SD-W314-001).

\subsection{TEST AND ACCEPTANCE}

The Project W-314 24l-AN-A Valve Pit modification STEP includes design verifications performed by various methods including engineering analysis, Vendor data review, FATs and CATs performed in accordance with Procurement/Construction Specifications, drawings, and system functional tests performed in accordance with ATPS and OTPS. These verifications and tests will be documented in the $W$-3I4 Project 24l-AN-A Requirements Verification Report (RVR), HNF-1534, Rev.0, and in specific test documentation (e.g., ATRs, OTRs, CWP...etc.). In addition, approved calibration and functional test procedures will be performed in preparation for the above listed tests.

\subsection{Validation and Verification}

All design requirements listed in the Project Development Specifications are formally reviewed by analysis and examination for project compliance. Commercial grade equipment utilized in Safety Class applications, will be qual ified per appropriate procedures and programs. Equipment used in non-safety class applications will be verified using vendor data to ensure the design requirements of the application are met. These reviews are documented in the 241-AN-A Valve Pit RVR. This report is listed in the reference section of this document. 


\subsection{Commercial Grade Item Dedication}

The following table lists the testing that will be performed to satisfy the Commercial Grade Item Dedication requirements for the modification of 241-AN-A Valve Pit. Note that acceptance criteria are included in the individual drawings, specifications, procedures, and CWPS.

TABLE 7-2

\begin{tabular}{|c|c|c|c|c|c|}
\hline \multicolumn{6}{|c|}{ 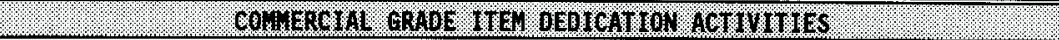 } \\
\hline TEST NUMBER & $\begin{array}{l}\text { TEST } \\
\text { NAME }\end{array}$ & $\begin{array}{l}\text { REFERENCE } \\
\text { DOCUMENT }\end{array}$ & LOCATION & TEST DOCUMENT & INTERDEPENDENCIES \\
\hline 1.4.C.A.CG.1 & $\begin{array}{l}\text { Rebar Tensile Test } \\
\text { and Physical } \\
\text { Inspection }\end{array}$ & $\begin{array}{l}\text { W-314-C1, section } \\
03300,1.5 .2 .2\end{array}$ & Shop & & $\begin{array}{l}\text { Test reports must be in place prior } \\
\text { to installing any Rebar. }\end{array}$ \\
\hline 1.4.C.A.CG 2 & $\begin{array}{l}\text { Steel Plate, bar and } \\
\text { shape Tensile test } \\
\text { and physical } \\
\text { Inspection }\end{array}$ & $\begin{array}{l}\text { W-314-C1, section } \\
05500,1.3 .2 .1\end{array}$ & Shop & & $\begin{array}{l}\text { Test reports must be in place prior } \\
\text { to installing any items for which } \\
\text { testing was required. }\end{array}$ \\
\hline 1.4.C.A.CG.3 & $\begin{array}{l}\text { Commercial Grade } \\
\text { Dedication for } \\
\text { instrumentation }\end{array}$ & $\begin{array}{l}\text { W-314-C1, section } \\
\text { 13440, 2.3.2; RVR } \\
\text { App.C 3.3.5.1 }\end{array}$ & Shop & & $\begin{array}{l}\text { Tests must be documented as } \\
\text { acceptable prior to installing any } \\
\text { components in field locations }\end{array}$ \\
\hline 1.4.C.A.CG.4 & $\begin{array}{l}\text { Commercial Grade } \\
\text { Dedication for Piping } \\
\text { Materials, verify pipe } \\
\text { size }\end{array}$ & $\begin{array}{l}\text { W-314-C1, section } \\
15493,1.2 .3 .1 \text { and } \\
2.4: \text { RVR App.A } \\
3.2 .2 .6\end{array}$ & Shop & & $\begin{array}{l}\text { Test Reports must be accepted } \\
\text { prior to fabrication of piping spools. }\end{array}$ \\
\hline
\end{tabular}

\subsection{Factory Acceptance Testing}

The following table lists the FATs that will be performed to modify 241-AN-A Valve Pit. Note that acceptance criteria are included in the individual drawings, specifications, procedures, and CWPs.

TABLE 7-3

\begin{tabular}{|c|c|c|c|c|c|}
\hline \multicolumn{6}{|c|}{ 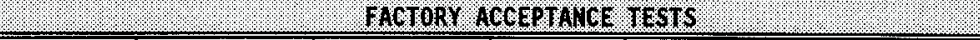 } \\
\hline TEST NUMBER & $\begin{array}{l}\text { TEST } \\
\text { NAME }\end{array}$ & $\begin{array}{l}\text { REFERENCE } \\
\text { DOCUMENT }\end{array}$ & LOCATION & TEST DOCUMENT & INTERDEPENDENCIES \\
\hline $1,4, C, A, F, 1$ & $\begin{array}{l}\text { Hydro and Leak } \\
\text { tests }\end{array}$ & $\begin{array}{l}\text { W-314-P1, section } \\
\text { 3.9 }\end{array}$ & Factory & & Prior to shipping \\
\hline 1.4.C.A.F. 2 & $\begin{array}{l}\text { Impact Wrench } \\
\text { Assembly }\end{array}$ & $\begin{array}{l}\text { W-314-P2, section } \\
3.6\end{array}$ & Factory & & Prior to shipping \\
\hline
\end{tabular}




\subsection{Construction Acceptance Testing}

The table below lists the construction tests performed during fabrication, modification and installation to verify functionality of structures, systems and components following installation. AI inspection activities performed during construction are referenced in "ACCEPTANCE INSPECTION PLAN", W-314-C1-1, REV. B. Note that acceptance criteria are included in the individual test drawings, specifications, procedures, and CWPs.

\section{TABLE 7-4}

\begin{tabular}{|c|c|c|c|c|c|}
\hline & & GOA SRO & 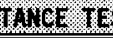 & & \\
\hline TEST NUMBER & TEST & REFERENCE DOCUMENT & LOCATION & $\begin{array}{l}\text { TEST } \\
\text { DOCUMENT }\end{array}$ & INTERDEPENDENCIES \\
\hline 1.4.C.A.C.1 & In-Place Density Test & $\begin{array}{l}\text { W-314-C1, section } 02220 \text {. } \\
3.1 .6 .2\end{array}$ & Field & CWP & $\begin{array}{l}\text { Required prior to excavation for all } \\
\text { excavations }\end{array}$ \\
\hline 1.4.C.A.C. 2 & Backfill Density Test & $\begin{array}{l}\text { W-314-C1, section } 02220,3.3 \text {, } \\
\text { 3.4, 3.6.1; RVR App.A 3.3.1.6, } \\
\text { 3.3.1.7 }\end{array}$ & Field & CWP & During \& after backfill \\
\hline 1.4.C.A.C.3 & Concrete Slump Test & $\begin{array}{l}\text { W.314-Ct, section } 03300, \\
\text { 1.5.2.1, 3.4.1; RVR App.A } \\
\text { 3.3.14, App.B 3.3.4.2 }\end{array}$ & Field & CWP & During concrete placement \\
\hline 1.4.C.A.C. 4 & Concrete Air Content Test & $\begin{array}{l}\text { W-314-C1, section } 03300 \text {, } \\
\text { 1.5.2.1; RVR App.A } 3.3 .14 \text {, } \\
\text { App.B 3.3.4.2 }\end{array}$ & Field & CWP & During concrete placement \\
\hline 1.4.C.A.C.5 & Concrete Test Cylinders & $\begin{array}{l}\text { W-314-C1, section 03300, } \\
\text { 1.5.2.1: RVR App.A 3.3.14, } \\
\text { App.B 3.3.4.2 }\end{array}$ & Field, Lab & $\begin{array}{l}\text { Testing } \\
\text { Agency Report }\end{array}$ & $\begin{array}{l}\text { Cast cylinders during concrete } \\
\text { placement }\end{array}$ \\
\hline 1.4.C.A.C. 6 & $\begin{array}{l}\text { Concrete Preplacement } \\
\text { Inspections }\end{array}$ & $\begin{array}{l}\text { W-314-C1, section 03300, 3.1, } \\
\text { 3.2.1, 3.2.2.1, \& 3.4.2: RVR } \\
\text { App.B 3.7.3.2.4 }\end{array}$ & Field & $\begin{array}{l}\text { CWP \& } \\
\text { Concrete pour } \\
\text { slip }\end{array}$ & Prior to Placing Concrete \\
\hline 1.4.C.A.C. 7 & $\begin{array}{l}\text { Concrete Placement } \\
\text { inspections }\end{array}$ & $\begin{array}{l}\text { W-314-C1, section } 03300,3.2 \& \\
3.4 .2\end{array}$ & Field & CWP & During concrete placement \\
\hline 1.4.C.A.C. 8 & $\begin{array}{l}\text { Concrete Post-Placement } \\
\text { inspections }\end{array}$ & $\begin{array}{l}\text { W-314-C1, section 03300, 3.2, } \\
\text { 3.3\& } 3.4 .2\end{array}$ & Field & CWP & Prior to Cover block Installation. \\
\hline 1.4.C.A.C. 9 & $\begin{array}{l}\text { Expansion Anchor } \\
\text { Inspections }\end{array}$ & W-314-C1, section 05055, 3.4.1 & Field & CWP & During anchor installation \\
\hline 1.4.C.A.C. 10 & $\begin{array}{l}\text { Structural Weld } \\
\text { Examinations }\end{array}$ & W-314-C1, section $05500,3.4 .1$ & Shop, Field & CWP & Prior to coating any structural pieces. \\
\hline 1.4.C.A.C. 11 & $\begin{array}{l}\text { Special Protective Coating } \\
\text { Surface Preparation } \\
\text { Inspection }\end{array}$ & $\begin{array}{l}\text { W-314-C1, section 09855, 3.2.2 } \\
\text { \& 3.4.2; RVR App.D 3.3.1.2.1 }\end{array}$ & Shop, Field & CWP & Prior to application of coatings \\
\hline 1.4.C.A.C. 12 & $\begin{array}{l}\text { Special Protective Coating } \\
\text { Application }\end{array}$ & $\begin{array}{l}\text { W-314-C1, section 09855, } \\
\text { 3.4.1; RVR App.D 3.2.2.1, } \\
\text { 3.2.2.2, 3.2.2.8, 3.2.3, 3.2.4.1 }\end{array}$ & Shop. Field & CWP & During application of each coat \\
\hline 1.4.C.A.C.13 & $\begin{array}{l}\text { Cover Block Painting } \\
\text { Diagrams }\end{array}$ & $\begin{array}{l}\text { W-314-C1, section 09855, 3.3.5 } \\
\text { \& 3.4.2; RVR App.B 3.7.3.2.5 }\end{array}$ & Shop & CWP & $\begin{array}{l}\text { Verify painting diagram matches } \\
\text { information given in drawing } \mathrm{s} \\
\text { referenced in section } 3.3 .6 \text {. }\end{array}$ \\
\hline 1.4.C.A.C.14 & Wiring Continuity & $\begin{array}{l}\text { W-314-C1, section } 13440, \\
\text { 3.2.1.1 \& section } 16400,3.3 .1 .1\end{array}$ & Shop, Field & CWP & Complete prior to performing ATP. \\
\hline 1.4.C.A.C. 15 & $\begin{array}{l}\text { Shielding Resistance to } \\
\text { Ground }\end{array}$ & $\begin{array}{l}\text { W-314-C1, section } 13440 \\
3.2 .1 .2\end{array}$ & Field & CWP & Complete prior to performing ATP \\
\hline 1.4.C.A.C. 16 & Voltage Verification & $\begin{array}{l}\text { W-314-C1, section } 16400 \text {, } \\
\text { 3.3.1.2 }\end{array}$ & Field & CWP & Prior to energizing equipment. \\
\hline
\end{tabular}


HNF-1733, REV. 0

\begin{tabular}{|c|c|c|c|c|c|}
\hline \multicolumn{6}{|c|}{ 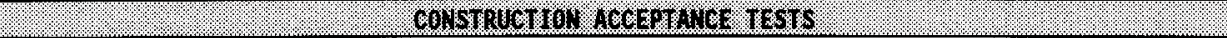 } \\
\hline TEST NUMBER & TEST: & REFERENCE DOCUMENT & LOCATION & $\begin{array}{l}\text { TEST } \\
\text { DOCUMENT }\end{array}$ & NTERDEPENDENCIES \\
\hline 1.4.C.A.C. 17 & $\begin{array}{l}\text { Holiday Testing of Piping } \\
\text { Protective Coatings }\end{array}$ & $\begin{array}{l}\text { W-314-C1, section } 15493 \text {. } \\
3.1 .6 .3\end{array}$ & Field & CWP & Prior to Backilili. \\
\hline 1.4.C.A.C. 18 & $\begin{array}{l}\text { Welding Non-Destructive } \\
\text { Examination }\end{array}$ & $\begin{array}{l}\text { W-314-C1, section } 15493,3.2 .1 \\
\text { \&. Appendix A, 3.3 \& 4.1; RVR } \\
\text { App. A 3.3.1.5, 3.3.4, App. B } \\
\text { 3.3.4.1 }\end{array}$ & Shop, Field & CWP & Prior to any form of pressure testing. \\
\hline 1.4.C.A.C. 19 & Piping Cleanliness Inspection & $\begin{array}{l}\text { W-314-C1, section } 15493 \text {, } \\
\text { 3.2.3.5; RVR App. A } 3.3 .4\end{array}$ & Shop Field & CWP & Prior to any form of pressure testing \\
\hline 1.4.C.A.C. 20 & Piping Pressure Tests & $\begin{array}{l}\text { W-314-C1, section } 15493 \\
\text { 3.2.4; RVR App. A 3.3.1.2 }\end{array}$ & Shop & CWP & $\begin{array}{l}\text { Test Nozzle spools prior to installation } \\
\text { in the field. }\end{array}$ \\
\hline 1.4.C.A.C. 21 & Jumper Pressure Tests & $\begin{array}{l}\text { W-314-C1, section } 15493 \\
\text { Appendix A, 3.6 \& 4.3; RVR } \\
\text { App. B 3.2.1.2.5 }\end{array}$ & Shop & CWP & $\begin{array}{l}\text { Test all Jumpers prior to installation in } \\
\text { the value pit }\end{array}$ \\
\hline 1.4.C.A.C. 22 & $\begin{array}{l}\text { Jumper Fabrication } \\
\text { Inspections }\end{array}$ & $\begin{array}{l}\text { W-314-C1, section 15493, } \\
\text { Appendix A, } 4.2\end{array}$ & Shop & CWP & Prior to shop hydro \\
\hline 1.4.C.A.C. 23 & Jumper In service Leak Test & $\begin{array}{l}\text { HNF-SD-B1O-001, section } \\
\text { 5.3.2.18, TSR-006, AC 5.12.2.a }\end{array}$ & Field & $\begin{array}{l}\text { CWP and } \\
\text { Leak Test } \\
\text { Procedure }\end{array}$ & $\begin{array}{l}\text { Installation of all jumpers and required } \\
\text { changes at associated tank central } \\
\text { pump pits. }\end{array}$ \\
\hline 1.4.C.A.C. 24 & $\begin{array}{l}\text { Leak Detector/Drain Plug } \\
\text { Mock-up }\end{array}$ & $\begin{array}{l}\text { HNF-SD-W314-PDS-003, } \\
\text { section 4.1.2; RVR App. A } \\
3.7 .2 .1 .1 .3,3.7 .2 .1 .1 .4 \text {. } \\
3.7 .2 .1 .1 .5, \text { App. C } 3.2 .1 .1 .4 \text {, } \\
3.2 .1 .1 .5,3.2 .1 .1 .6,3.2 .1 .2 .1 \\
3.2 .1 .2 .2,3.2 .1 .2 .3,3.2 .1 .3 .1 \\
3.2 .1 .3 .2,3.2 .1 .3 .3,3.2 .2 .2 \\
3.2,4.3\end{array}$ & & & $\begin{array}{l}\text { Mockup testing must be completed and } \\
\text { results approved prior to installation in } \\
\text { the field. }\end{array}$ \\
\hline 1.4.C.A.C. 25 & $\begin{array}{l}\text { Elegtrical \& Instrumentation } \\
\text { Configuration \& Code } \\
\text { Compliance }\end{array}$ & $\begin{array}{l}\text { RVR App. A 3.3.1.10, 3.3.1.11, } \\
\text { 3.3.1.12, App. С 3.3.1.1, 3.3.1.2 }\end{array}$ & Field & CWP & Prior to perforrning OTP \\
\hline 1.4.C.A.C. 26 & $\begin{array}{l}\text { Piping Configuration } \\
\text { Verification }\end{array}$ & $\begin{array}{l}\text { RVR App. A 3.2.2.1, 3.2.2.7. } \\
\text { 3.2.2.8, 3.2.2.9, 3.2.2.10 } \\
3.3 .1 .8\end{array}$ & Shop, Field & CWP & Prior to installation of the wall nozzles \\
\hline 1.4.C.A.C.27 & Cathodic Protection & $\begin{array}{l}\text { W-314-C1, section 16640, } \\
\text { 3.3.1, 3.3.2 }\end{array}$ & Field & CWP & $\begin{array}{l}\text { After nozzle installation and prior to } \\
\text { application of protective coating on pipe }\end{array}$ \\
\hline 1.4.C.A.C. 28 & Jumper Fit Up \& Installation & TWRS Operations Requirement & Field & CWP & $\begin{array}{l}\text { TWRS Operations witness jumper fit } \\
\text { up, balance, \& installation }\end{array}$ \\
\hline 1.4.C.A.C.29 & $\begin{array}{l}\text { Cover Block Fit Up \& } \\
\text { Installation }\end{array}$ & TWRS Operations Requirement & Field & CWP & $\begin{array}{l}\text { TWRS Operations witness jumper fit } \\
\text { up, balance, \& installation }\end{array}$ \\
\hline 1.4.C.A.C. 30 & $\begin{array}{l}\text { Load test Cover Block lifting } \\
\text { bails \& Lifting bail Magnetic } \\
\text { Particle Test }\end{array}$ & W-314-C1, 03300, section 3.2 .5 & Shop & CWP & $\begin{array}{l}\text { Prior to Special Protective Coating } \\
\text { Application/Installation. Lifting bail } \\
\text { Mag. Particle test must be performed } \\
\text { AFTER load test. }\end{array}$ \\
\hline 1.4.C.A.C.31 & $\begin{array}{l}\text { Jumper support leg } \\
\text { adjustment }\end{array}$ & $\begin{array}{l}\text { H-14-100948, Note } 7 \\
\text { H-14-100949, SH1, Note } 7 \\
\text { H-14-100950, SH1, Note 7 } \\
\text { H-14-100952, SH1, Note } 7 \\
\text { H-14-100953, SH1, Note } 7\end{array}$ & Field & CWP & Prior to Cover Block installation \\
\hline 1.4.C.A.C.32 & $\begin{array}{l}\text { Final Special Protective } \\
\text { Coating inspection of pit \& } \\
\text { cover block }\end{array}$ & $\begin{array}{l}\text { W-314 Project Management } \\
\text { requirement }\end{array}$ & Field & CWP & Prior to installation of caver block \\
\hline
\end{tabular}


HNF-1733, REV. 0

\subsection{Acceptance Testing}

Acceptance testing will be conducted by utilizing ATPs. ATPs are separate "stand alone" documents which obtain their requirements from the applicable PDS, drawings, and vendor data. ATPs will ensure that the SSCs are fabricated and/or installed properly per the design. Detailed ATPS will be required after the CAT functional checks and/or inspections are completed and, will validate overall SSC installation as a whole. The acceptance criteria shall be specified in the test procedures and/or on approved drawings and specifications. These tests will be performed on components in their installed condition but may not exercise the entire system as an operational unit. The table below describes the ATPs that will be performed for 24l-AN-A Valve Pit modification and upgrade acceptance testing. An Acceptance Test Report (ATR) will be generated when all test exceptions have been cleared and the test has been successfully completed. Note that acceptance criteria are included in the individual test document.

TABLE $7-5$

\begin{tabular}{|c|c|c|c|c|}
\hline \multicolumn{5}{|c|}{ 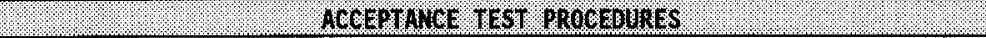 } \\
\hline $\begin{array}{l}\text { TEST } \\
\text { DOCUMENT }\end{array}$ & TEST DESCRIPTION & REFERENCE DOCUMENT & LOCATION & INTERDEPENDENCIES \\
\hline TBD & Plt Leak Detection & $\begin{array}{l}\text { HNF-SD-W314-PDS-003; } \\
\text { RVR App. A 3.3.2, } \\
3.7 .2 .1 .2 .1,3.7 .2 .1 .2 .2 \text {, } \\
3.7 .2 .1 .2 .3,3.7 .2 .1 .3 .1 \text {, } \\
3.7 .2 .1 .3 .2,3.7 .2 .1 .3 .3 \text {, } \\
\text { App. B } 3.3 .2, \text { App. C } \\
3.2 .2 .5,3.3 .2\end{array}$ & Field & Leak Detector installed and energized \\
\hline TBD & $\begin{array}{l}\text { Encasement Leak } \\
\text { Detection }\end{array}$ & $\begin{array}{l}\text { HNF-SD-W314-PDS-003; } \\
\text { RVR App. A 3.2.4.5, 3.3.2, } \\
\text { 3.7.2.1.2.1, 3.7.2.1.2.2, } \\
3.7 .2 .1 .2 .3,3.7 .2 .1 .3 .1, \\
3.7 .2 .1 .3 .2,3.7 .2 .1 .3 .3 \\
\text { App. B } 3.3 .2, \text { App. C } \\
3.2 .2 .5,3.3 .2\end{array}$ & Field & Leak Detector installed and energized \\
\hline TBD & Cathodic Protection & HNF-SD-W314-PDS-001 & Field & Prior to backfill \\
\hline TBD & $\begin{array}{l}\text { Valve Manifold and } \\
\text { Indicators (Position } \\
\text { Switches) }\end{array}$ & $\begin{array}{l}\text { HNF-SD-W314-PDS-002; } \\
\text { RVR App. B 3.7.2.1.1.4, } \\
3.7 .2 .2 .1,3.7 .2 .2 .3 \\
3.7 .2 .2 .4\end{array}$ & Field & $\begin{array}{l}\text { After equipment installation on cover } \\
\text { block }\end{array}$ \\
\hline TBD & $\begin{array}{l}\text { Impact Wrench } \\
\text { Assembly }\end{array}$ & $\begin{array}{l}\text { TWRS Operations } \\
\text { Requirement }\end{array}$ & $\begin{array}{l}\text { Shop or } \\
\text { Factory or } \\
\text { field }\end{array}$ & Prior to field jumper installation \\
\hline
\end{tabular}


HNF-1733, REV. 0

\subsection{Operational Testing}

Operational testing will be conducted by utilizing 0TPs. OTPs will be planned, scheduled, and conducted, on the installed SSCs, after completion and acceptance of all other test results. These detailed procedures shall determine if the installed SSCs meet the PDS functional requirements. Included in the OTP will be acceptance criteria to delineate pass/fail requirements. Operational testing is performed with actual plant equipment, operating procedures, and personnel. If an OTP has been generated but the testing of the corresponding SSC won't be performed for an extended period of time (e.g., a year or more), then the OTP must be reviewed again, prior to performance of the test, to ensure that it is still applicable. A Operational Test Report (OTR) will be generated when all test exceptions have been cleared and the test has been successfully completed. Testing of valve operators (with the vaives connected), drain plug and leak detectors will be conducted with these components in their normal operating positions. Test conditions will meet the required code, design and authorization basis (i.e., Basis for Interim 0peration, Technical Safety Requirements, and HNF-IP-1266, "TANK FARM OPERATIONS ADMINISTRATIVE CONTROLS") to verify that the system is functional and safe to operate. The following table describes the various sections of the OTP for 241-AN-A valve pit modifications. Note that acceptance criteria are included in the individual test document. 
HNF-1733, REV. 0

TABLE 7-6

\begin{tabular}{|c|c|c|c|c|c|}
\hline \multicolumn{6}{|c|}{ 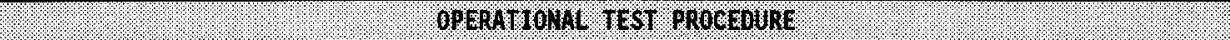 } \\
\hline Test Section & Test Description & $\begin{array}{l}\text { Reference } \\
\text { Document }\end{array}$ & Location & $\begin{array}{c}\text { Test } \\
\text { Document }\end{array}$ & Interdependencies \\
\hline TBD & $\begin{array}{l}\text { Architectural: } \\
\text { This section veriftes that all components are } \\
\text { physically in place per the drawings, that they have } \\
\text { been correctly labeled, painted, etc. Items included } \\
\text { are electrical boxes, cover blocks, shielding blocks, } \\
\text { valve operalors, position indicators, valve handles, } \\
\text { drain plug handles, and jumpers between cover } \\
\text { blocks and terminal boxes. Piping jumpers and } \\
\text { internal pit coating are specifically excluded from this } \\
\text { inspection as they will be verified as part of earlier } \\
\text { construction tests witnessed by operations. }\end{array}$ & $\begin{array}{l}\text { RVR App. A } \\
3.3 .3 .1, \text { App. B } \\
3.2 .1 .1 .1,3.2 .2 .1 \text {, } \\
3.2 .2 .2,3.3 .3 .1 \text {, } \\
3.7 .3 .1 .1 .1 \text {, } \\
3.7 .3 .2 .1 \text {, } \\
3.7 .3 .2 .3 \text {, App. C } \\
3.3 .3 .1\end{array}$ & Field & OTP-314-001 & All equipment in place, \\
\hline$T B D$ & $\begin{array}{l}\text { Valve Operation: } \\
\text { This section tests the operation of the valve } \\
\text { operators and valve position indication. Operators } \\
\text { are verified to be able to move the valves and } \\
\text { remote indication of the position switches at the } \\
\text { terminal box is verified for all positions of each value. } \\
\text { Verify functionality of valve locking devices. }\end{array}$ & & Field & OTP-314-001 & $\begin{array}{l}\text { All equipment in place. Locks and } \\
\text { tags in place to prevent inadvertent } \\
\text { transfer of waste. }\end{array}$ \\
\hline TBD & $\begin{array}{l}\text { Leak Detector and Drain plug Operation: } \\
\text { This section verifies the operation of the leak } \\
\text { detector and the drain plug by adding water to the pit } \\
\text { until the leak detector actuates the alarms at } 271-A N \\
\text { and all other appropriate locations, and actuates the } \\
\text { master pump shutdown. Acceptance criteria for } \\
\text { operation is the amount and rate of water that the } \\
\text { system should detect per the design verification } \\
\text { report. This detection test shall be repeated no less } \\
\text { than three (3) times). }\end{array}$ & $\begin{array}{l}\text { RVR App. A } \\
\text { 3.3.5. App. C } \\
\text { 3.2.4.4 }\end{array}$ & Field & OTP-314-001 & $\begin{array}{l}\text { All equipment in place, current } \\
\text { calibration of the leak detector and the } \\
\text { master pump shutdown system not in } \\
\text { alarm. }\end{array}$ \\
\hline
\end{tabular}

\subsection{1-AN-A Valve Pit Test Completion}

241-AN-A Valve Pit testing will be considered successfully completed when the TEST COMPLETION CHECKLIST (attached to the end of this STEP) is completely signed off. This checklist is a "stand alone" document that will be listed on the 241-AN-A Valve Pit ABU

(HNF-SD-W314-ABU-002) and included in the turnover of documents. This checklist will be completed by the W-314 Startup Manager or his designee. 
HNF-1733, REV. 0

\section{REFERENCES}

HNF-PRO-229, Technical Procedure Standard, Fluor Daniel Hanford Company, Richland, Washington.

HNF-PRO-242, Rev. 1, Engineering Drawing Requirements, Fluor Daniel Hanford Company, Richland, Washington.

HNF-PRO-440, Engineering Document Change Control Requirements, Fluor Daniel Hanford Company, Richland, Washington.

HNF-PRO-446, Testing Practices Requirements, Fluor Daniel Hanford Company, Richland, Washington.

HNF-PRO-572, Project Acceptance and Closeout, Fluor Daniel Hanford Company, Richland, Washington.

W-314-C1, Construction Specification for Tank Farm Restoration and Safe Operations AN Valve Pit Upgrades, Fluor Daniel Northwest, Inc., Richland, Washington.

W-314-P1, Procurement Specification Ball Valves AN Valve Pit Upgrades, Fluor Daniel Northwest, Inc., Richland, Washington.

W-314-P2, Procurement Specification Impact Wrench AN Va7ve Pit Upgrades, Fluor Daniel Northwest, Inc., Richland, Washington.

HNF-SD-W314-PDS-001, Rev. 0, Project Development Specification - Transfer Piping, Fluor Daniel Northwest, Inc., Richland, Washington.

HNF-SD-W314-PDS-002, Rev. 0, Project Development Specification for Valve Pit Manifold, Fluor Daniel Northwest, Inc., Richland, Washington.

HNF-SD-W314-PDS-003, Rev. 0, Project Development Specification for Pit Leak Detection, Fluor Daniel Northwest, Inc., Richland, Washington.

HNF-SD-W314-PDS-005, Rev. 0, Project Development Specification for Special Protective Coating, Fluor Daniel Northwest, Inc., Richland, Washington.

HNF-1534, Rev. 0, Requirements Verification Report for AN Valve Pit Upgrades (WBS 1.4.C), Fluor Daniel Northwest, Inc., Richland, Washington.

HNF-SD-W314-ABU-002, Rev. 0, AN-A VALVE PIT UPGRADE Acceptance For Beneficial Use, Rev. 0, Numatec Hanford Corporation, Richland, Washington.

HNF-SD-W314-TEP-001, Rev. 0, Test and Evaluation Plan for W-314 Tank Farm Restoration and Safe Operations, Numatec Hanford Corporation, Richland, Washington.

HNF-CM-3-5, Document Contro7 and Records Management, Fluor Daniel Hanford Company, Richland, Washington.

HNF-IP-0842, TWRS Administration, Fluor Daniel Hanford Company, Richland, Washington. 
HNF-1733, REV. 0

HNF-IP-1266, Tank Farm Operations Administrative Controls, Fluor Daniel Hanford Company, Richland, Washington.

HNF-SD-WM-BI0-001, Rev. F, TWRS Basis for Interim Operation, Fluor Daniel Hanford Corporation, Richland, Washington. 
HNF-1733, REV. 0

W-314-C1-1, Rev. B, Acceptance Inspection Plan, Fluor Daniel Hanford Corporation, Richland, Washington. 
HNF-1733, REV. 0

241-AN-A VALVE PIT TEST COMPLETION CHECKLIST

PAGE OF

\begin{tabular}{|c|c|c|c|c|c|}
\hline \multirow{2}{*}{ TEST NUMBER } & \multirow{2}{*}{$\begin{array}{l}\text { TEST } \\
\text { NAME }\end{array}$} & \multirow{2}{*}{$\begin{array}{c}\text { TEST } \\
\text { PROCEDUREDOCUMENT } \\
\text { NUMBEER }\end{array}$} & \multirow{2}{*}{$\begin{array}{l}\text { DATE TEST } \\
\text { COMPLETED }\end{array}$} & \multicolumn{2}{|c|}{ TEST COMPLETED AND RESULTS ACCEPTED (I.e, ALL EXCEPTIONS RESOLVED) } \\
\hline & & & & PRINT NAME. & SIGNATURE \\
\hline \multicolumn{6}{|c|}{ 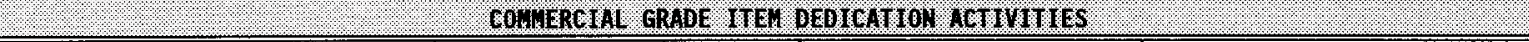 } \\
\hline 1.4.C.A.CG.1 & $\begin{array}{l}\text { Rebar Tensile Test and Physical } \\
\text { Inspection }\end{array}$ & & & & \\
\hline 1.4.C.A.CG.2 & $\begin{array}{l}\text { Steel Plate, bar and shape } \\
\text { Tensile test and physical } \\
\text { Inspection }\end{array}$ & & & & \\
\hline 1.4.C.A.CG.3 & $\begin{array}{l}\text { Commercial Grade Dedication for } \\
\text { instrumentation }\end{array}$ & & & & \\
\hline 1.4.C.A.CG.4 & $\begin{array}{l}\text { Commercial Grade Dedication for } \\
\text { Piping Materials }\end{array}$ & & & & \\
\hline \multicolumn{6}{|c|}{ RACTOAY ACCEPTAUCE TLSS } \\
\hline 1.4.C.A.F.1 & Hydro and Leak tests & & & & \\
\hline 1.4.C.A.F. 2 & Impact Wrench Assembly & & & & \\
\hline \multicolumn{6}{|c|}{ CONSTRUCT ION ACGEPTINCE. TESTS } \\
\hline 1.4.C.A.C.1 & In-Place Density Test & & & & \\
\hline 1.4.C.A.C. 2 & Backfill Density Test & & & & \\
\hline 1.4.C.A.C. 3 & Concrete Stump Test & & & & \\
\hline 1.4.C.A.C. 4 & Concrete Air Content Test & & & & \\
\hline 1.4.C.A.C.5 & Concrete Test Cylinders & & & & \\
\hline 1.4.C.A.C.6 & $\begin{array}{l}\text { Concrete Pre-placement } \\
\text { Inspections }\end{array}$ & & & & \\
\hline 1.4.C.A.C. 7 & Concrete Placement Inspections & & & & \\
\hline 1.4.C.A.C. 8 & $\begin{array}{l}\text { Concrete Post-Placement } \\
\text { inspections }\end{array}$ & & & & \\
\hline 1.4.C.A.C. 9 & Expansion Anchor Irspections & & & & \\
\hline 1.4.C.A.C. 10 & Structural Weld Examinations & & & & \\
\hline 1.4.C.A.C.11 & $\begin{array}{l}\text { Special Protective Coating } \\
\text { Surface Preparation Inspection }\end{array}$ & & & & \\
\hline
\end{tabular}


HNF-1733, REV. 0

241-AN-A VALVE PIT TEST COMPLETION CHECKLIST

PAGE OF

\begin{tabular}{|c|c|c|c|c|c|}
\hline \multirow{2}{*}{ TEST NUMBER } & \multirow{2}{*}{$\begin{array}{l}\text { TEST } \\
\text { NAME }\end{array}$} & \multirow{2}{*}{$\begin{array}{l}\text { TEST } \\
\text { PROCEDUREDOCUMENT } \\
\text { NUMBER }\end{array}$} & \multirow{2}{*}{$\begin{array}{l}\text { DATETEST } \\
\text { COMPLETED }\end{array}$} & \multicolumn{2}{|c|}{ TEST COMPLETED ANO RESULTS ACCEPTED (i.e, ALL EXCEPTIONS RESOLVED) } \\
\hline & & & & PRINT NAME & SIGNATURE \\
\hline 1.4.C.A.C. 12 & $\begin{array}{l}\text { Special Protective Coating } \\
\text { Application }\end{array}$ & & & & \\
\hline 1.4.C.A.C.13 & Cover Block Painting Diagrams & & & & \\
\hline 1.4.C.A.C.14 & Wiring Continuity & & & & \\
\hline 1.4.C.A.C. 15 & Shielding Resistance to Ground & & & & \\
\hline 1.4.C.A.C. 16 & Voltage Verification & & & & \\
\hline 1.4.C.A.C.17 & $\begin{array}{l}\text { Holiday Testing of Piping } \\
\text { Protective Coatings }\end{array}$ & & & & \\
\hline 1.4.C.A.C. 18 & $\begin{array}{l}\text { Welding Non-Destructive } \\
\text { Examination }\end{array}$ & & & & \\
\hline 1.4.C.A.C.19 & Piping Cleanliness Inspection & & & & \\
\hline 1.4.C.A.C. 20 & Piping Pressure Tests & & & & \\
\hline 1.4.C.A.C. 21 & Jumper Pressure Tests & & & & \\
\hline 1.4.C.A.C.22 & Jumper Fabrication Inspections & & & & \\
\hline 1.4.C.A.C. 23 & Jumper In senvice Leak Test & & & & \\
\hline 1.4.C.A.C. 24 & Leak Detector/Drain Plug Mockup & & & & \\
\hline 1.4.C.A.C. 25 & $\begin{array}{l}\text { Electrical \& Instrumentation } \\
\text { Configuration \& Code } \\
\text { Compliance }\end{array}$ & & & & \\
\hline 1.4.C.A.C. 26 & Piping Configuration Verification & & & & \\
\hline 1.4.C.A.C. 27 & Cathodic Profection & & & & \\
\hline 1.4.C.A.C. 28 & Jumper Fit Up \& Installation & & & & \\
\hline 1.4.C.A.C. 29 & Cover Block Fit Up \& Installation & & & & \\
\hline 1.4.C.A.C. 30 & $\begin{array}{l}\text { Load test Cover Block lifting bails } \\
\& \text { Lifting bail Magnetic Particle } \\
\text { Test }\end{array}$ & & & & \\
\hline 1.4.C.A.C.31 & Jumper support leg adjustment & & & & \\
\hline 1.4.C.A.C.32 & $\begin{array}{l}\text { Final Special Protective Coating } \\
\text { inspection of pit \& cover black }\end{array}$ & & & & \\
\hline
\end{tabular}


HNF-1733, REV. 0

241-AN-A VALVE PIT TEST COMPLETION CHECKLIST

PAGE OF

\begin{tabular}{|c|c|c|c|c|c|}
\hline \multirow{2}{*}{ TEST NUMBER } & \multirow{2}{*}{$\begin{array}{l}\text { TEST } \\
\text { NAME }\end{array}$} & \multirow{2}{*}{$\begin{array}{l}\text { TEST } \\
\text { PROCEDUREDOCUMENT } \\
\text { NUMBER }\end{array}$} & \multirow{2}{*}{$\begin{array}{l}\text { DATE TEST } \\
\text { COMPLETED } \\
\end{array}$} & \multicolumn{2}{|c|}{ EST COMPLETED AND RESULTS ACCEPTED (I.E., ALL EXCEPTIONS RESOLVED) } \\
\hline & & & & PRINT NAME & SIGNATURE \\
\hline \multicolumn{6}{|c|}{ ACOEPIMLE ICST PROCEBURES } \\
\hline TBD & Pit Leak Detection & & & & \\
\hline TBD & Encasement Leak Detection & & & & \\
\hline TBD & Cathodic Protection & & & & \\
\hline TBD & $\begin{array}{l}\text { Valve Manifold and Indicators } \\
\text { (Position Switches) }\end{array}$ & & & & \\
\hline TBD & Impact Wrench Assembly & & & & \\
\hline \multicolumn{6}{|c|}{ OPERAT IONAL IEST PROCEOURE. } \\
\hline & 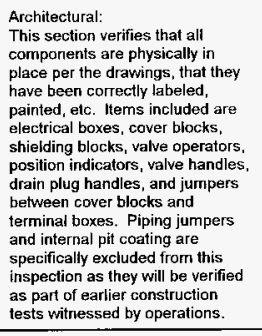 & & & & \\
\hline & $\begin{array}{l}\text { Valve Operation: } \\
\text { This section tests the operation of } \\
\text { the valve operators and valve } \\
\text { position indication. Operators are } \\
\text { verified to be able to move the } \\
\text { valves and remote indication of } \\
\text { the position switches at the } \\
\text { terminal box is verified for all } \\
\text { positions of each valve. Verify } \\
\text { functionality of valve locking } \\
\text { devices. }\end{array}$ & & & & \\
\hline
\end{tabular}


HNF-1733, REV. 0

241-AN-A VALVE PIT TEST COMPLETION CHECKLIST

PAGE

OF

\begin{tabular}{|c|c|c|c|c|c|}
\hline \multirow{2}{*}{ TEST NUMBER } & \multirow{2}{*}{$\begin{array}{c}\text { TEST } \\
\text { NAME }\end{array}$} & \multirow{2}{*}{$\begin{array}{c}\text { TEST } \\
\text { PROCEDUREDDOCUMENT } \\
\text { NUMBER } \\
\end{array}$} & \multirow{2}{*}{$\begin{array}{l}\text { DATETEST } \\
\text { COMPLETED }\end{array}$} & \multicolumn{2}{|c|}{ TEST COMPLETED AND RESULTS ACCEPTED (i.e, ALL EXCEPTIONS RESOLVED) } \\
\hline & & & & PRINT NAME. & SIGNATURE \\
\hline & $\begin{array}{l}\text { Leak Detector and Drain plug } \\
\text { Operation: } \\
\text { This section verifies the operation } \\
\text { of the leak detector and the drain } \\
\text { plug by adding water to the pit } \\
\text { until the leak detector actuates } \\
\text { the alarms at } 271 \text {-AN and all } \\
\text { other appropriate locations, and } \\
\text { actuates the master pump } \\
\text { shutdown. Acceptance criteria for } \\
\text { operation is the amount and rate } \\
\text { of water that the system should } \\
\text { detect per the design verification } \\
\text { report. This detection test shall } \\
\text { be repeated no less than three } \\
\text { (3) times). }\end{array}$ & & & & \\
\hline
\end{tabular}




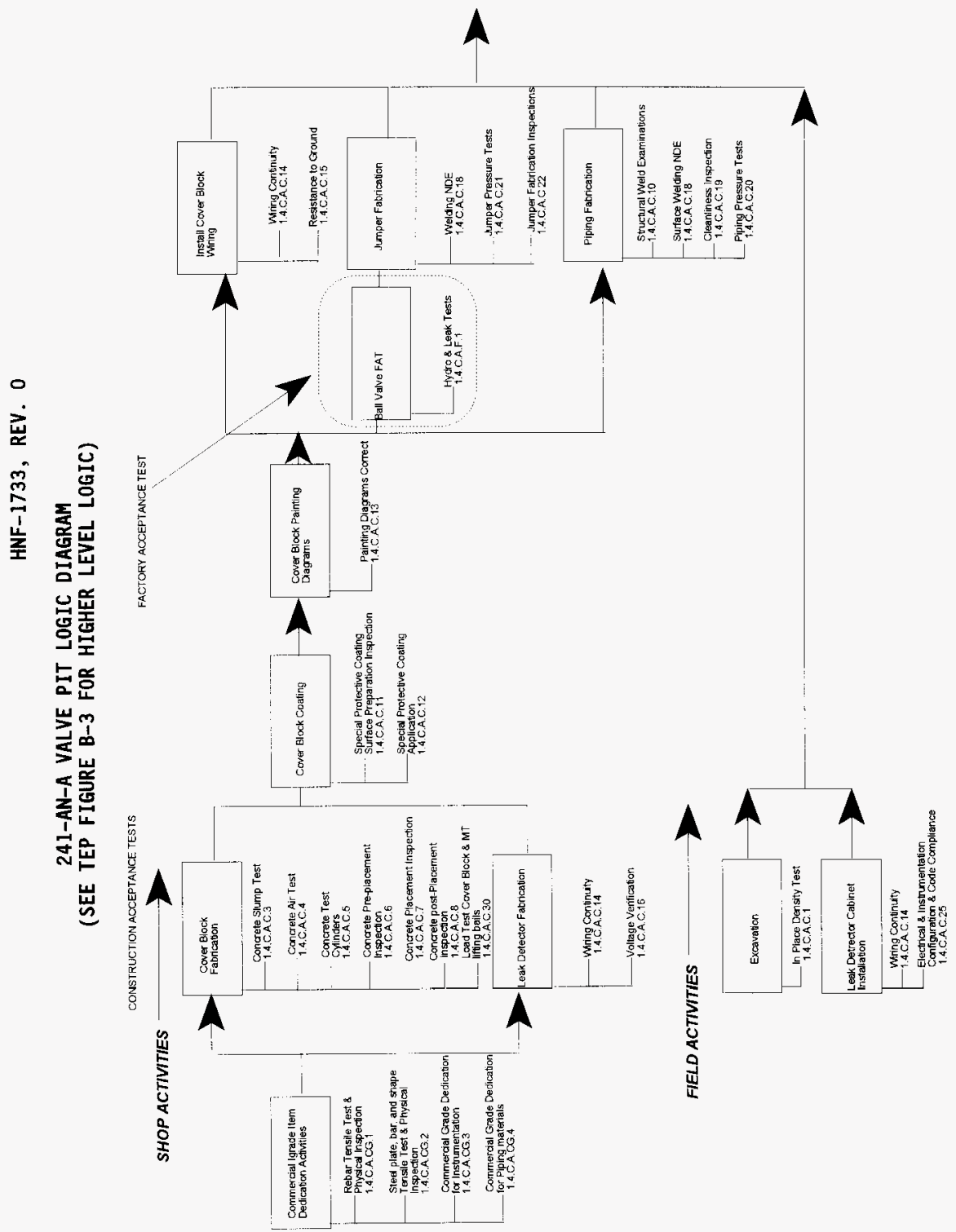


HNF-1733, REV. 0

241-AN-A VALVE PIT LOGIC DIAGRAM [cont.]

(SEE TEP FIGURE B-3 FOR HIGHER LEVEL LOGIC)

CONSTRUCTION AOCEPTANCE TES TS ; cont:

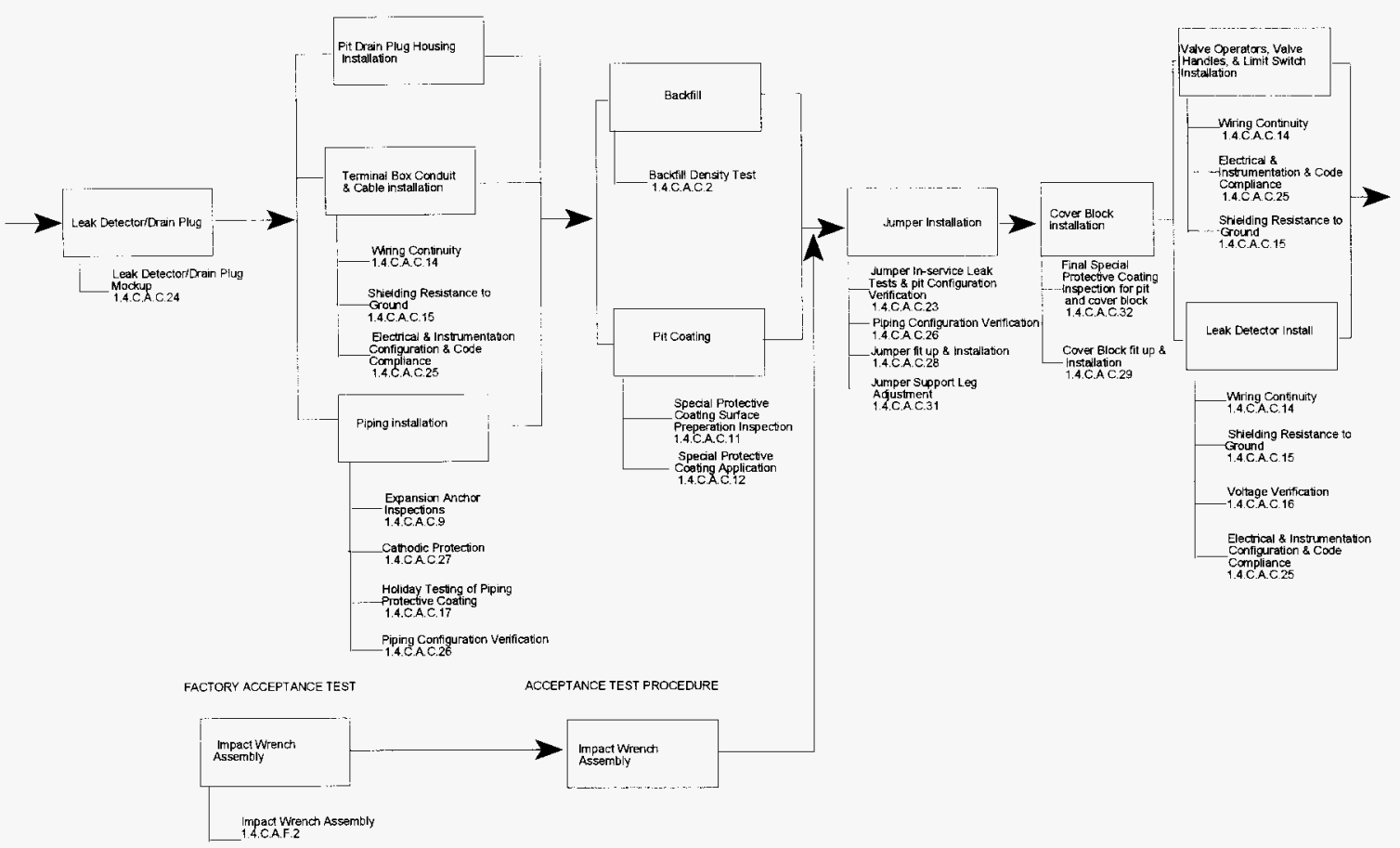


HNF-1733, REV. 0

241-AN-A VALVE PIT LOGIC DIAGRAM [cont.]

(SEE TEP FIGURE B-3 FOR HIGHER LEVEL LOGIC)

ACCEPTANCE TEST PROCEDURES

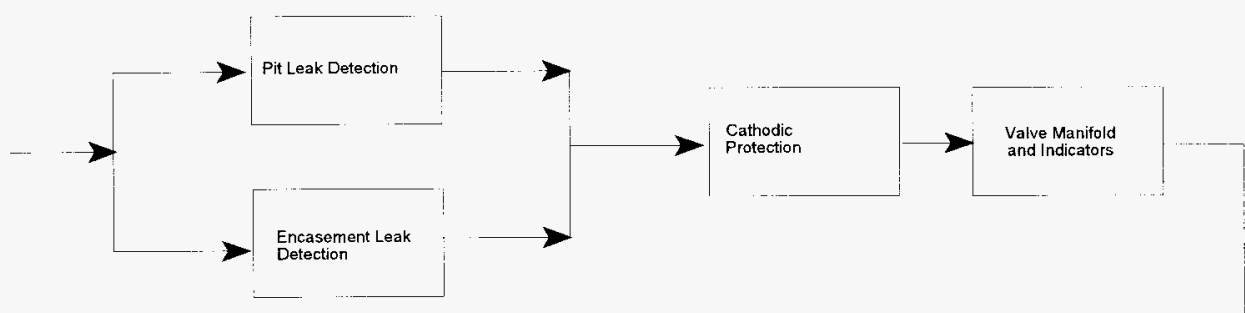

OPERATIONAL TEST PROCEDURE

All Equipment In Place

(Construction Complete)

Pit Drain Plug in Operate

Pit Drain
Position

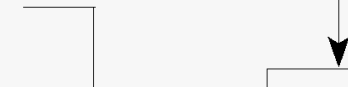

Lock \& Tags on Pumps

Leak Detection In Service

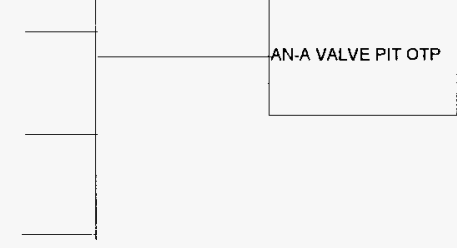




\section{DISTRIBUTION SHEET}

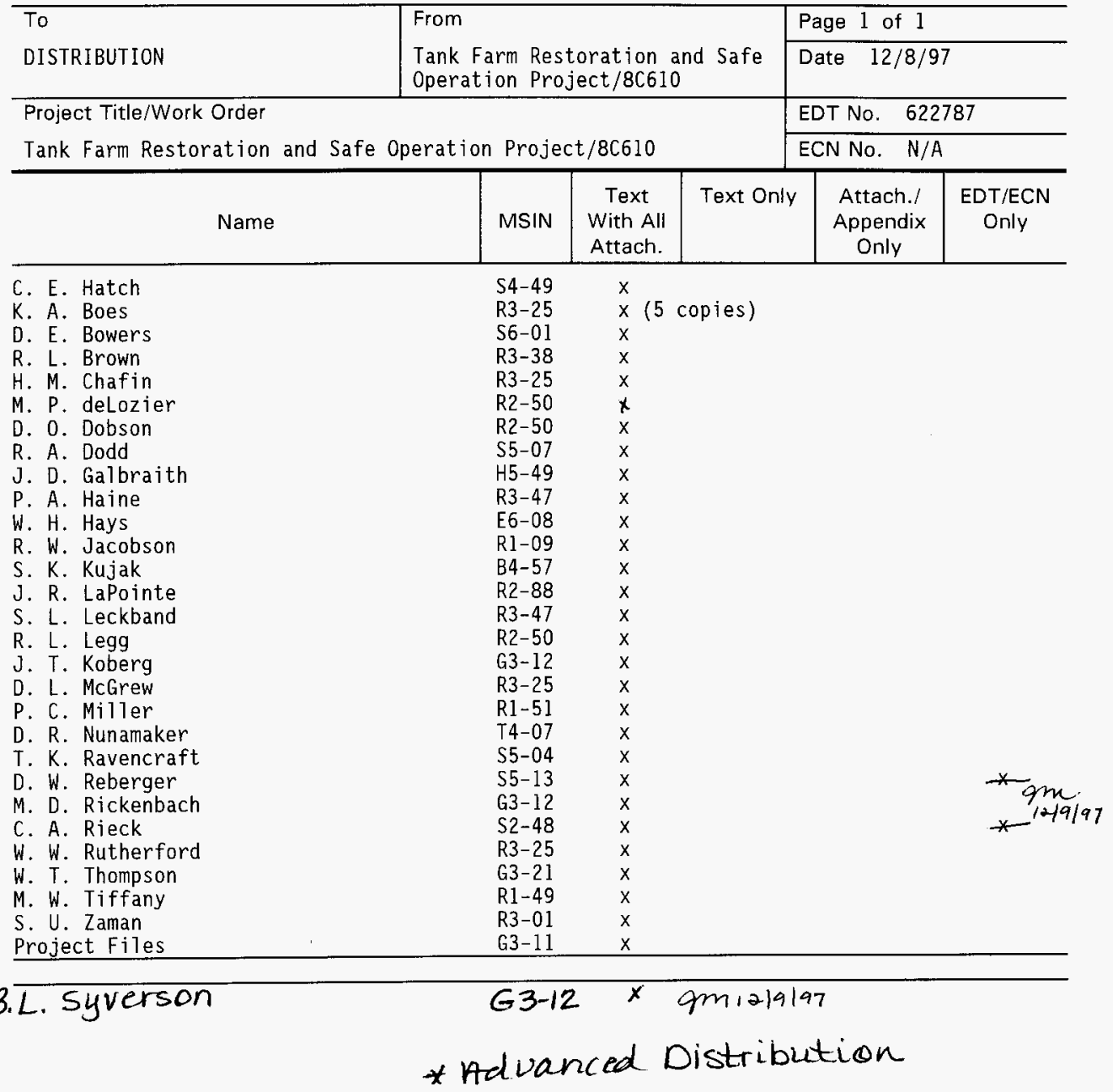

